\title{
Observations outside Radio Astronomy
}

\section{Olaf Scholten}

KVI-Center for Advanced Radiation Technology, Zernikelaan 25, 9747AA Groningen, The Netherlands E-mail: scholten@kvi.nl

\section{Jose Bacelar}

ASML Netherlands B.V., De Run 6501, 5504 DR Veldhoven, The Netherlands

\section{Stijn Buitink}

Astrophysical Institute, VUB, Pleinlaan 2, 1050 Brussel, Belgium

E-mail: Stijn.Buitink@vub.be

\section{Heino Falcke}

ASTRON, Fac. NWI, Radbout University Nijmegen, Postbus 9010, 6500 GL Nijmegen, The Netherlands E-mail: H.Falcke@astro.ru.nl

\section{Hans van der Marel}

ASTRON

Oude Hoogeveensedijk 4, 7991 PD Dwingeloo, The Netherlands

E-mail:marel@astron.nl

\section{Hans van der Marel}

Fac. CITG, Delft Technical University

Postbox 5048, 2600GA Delft, The Netherlands

E-mail:

H.vanderMarel@tudelft.nl

\section{Roland Klees}

Fac. CITG, Delft Technical University, Postbox 5048, 2600GA Delft, The Netherlands E-mail: R.Klees@tudelft.nl

\section{Arnold van Ardenne}

ASTRON

Oude Hoogeveensedijk 4, 7991 PD Dwingeloo, The Netherlands

E-mail: ardenne@astron.nl

\section{Maaijke Mevius}

ASTRON

Oude Hoogeveensedijk 4, 7991 PD Dwingeloo, The Netherlands

E-mail: mevius@astron.nl 


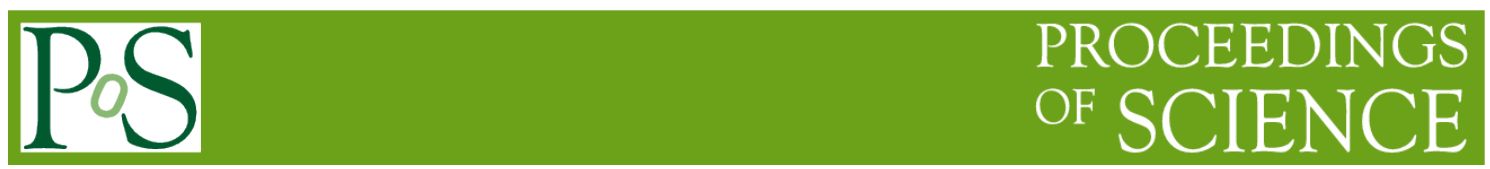

\section{Mark Bentum}

ASTRON

Oude Hoogeveensedijk 4, 7991 PD Dwingeloo, The Netherlands

E-mail: bentum@astron.nl

\section{Koos Kegel}

ASTRON

Oude Hoogeveensedijk 4, 7991 PD Dwingeloo, The Netherlands

E-mail: kegel@astron.nl

\section{Andre Bos}

Science and Technology B.V., Olaf Palmestraat 14, 2616LR Delft, The Netherlands E-mail: bos@stcorp.nl

50 Years Westerbork Radio Observatory, A Continuing Journey to Discoveries and Innovations Richard Strom, Arnold van Ardenne, Steve Torchinsky (eds) 


\section{Observations outside Radio Astronomy}

\section{Chapter 17.1 The NuMoon experiment at the WSRT}

Olaf Scholten', Jose Bacelar², Stijn Buitink ${ }^{3}$ and Heino Falcke ${ }^{4,5}$

KVI-CART, Univ. of Groningen, The Netherlands ${ }^{2}$ ASML Netherlands BV, The Netherlands ${ }^{3}$ Astrophyscal Institute, VUB, Belgium ${ }^{4}$ IMAPP, Univ. of Nijmegen, The Netherlands ${ }^{5}$ ASTRON, The Netherlands
I

t was at a party at ASTRON in 2005 that Ger de Bruyn suggested to some of us that it should be possible to use the WSRT to measure ultra-high energy (UHE) neutrinos.

Neutrinos are particles that interact only through the weak interactions and thus they travel basically un-attenuated over cosmological distances along straight-line trajectories. They are expected to be produced when high energy cosmic rays interact with matter. However while cosmic rays travel finite distances and furthermore travel along curved trajectories due to magnetic fields, the neutrinos always point back to their source. To detect neutrinos requires large detectors to compensate for their small interaction cross section. High energy neutrinos, with energies up to $10^{16} \mathrm{eV}$, have been observed with IceCube [Aartsen+ (2015)] a detector of cubic kilometer size. Since the flux is expected to drop steeply with energy we need much larger detectors to detect ultra-high energy $\left(E>10^{20} \mathrm{eV}\right)$ neutrinos. They are of much interest since they are the messengers of the most energetic processes in the universe, such as Blazars [IceCube (2018)], supermassive black-hole mergers, or may result from the decay of massive particles that are relics from the Big Bang.

The basic idea of the UHE neutrino observations, first proposed by Dagesamanskii \& Zheleznyk (1989), is that we have a really big detector in our back-yard, the moon. UHE neutrinos hitting the moon will form a cascade of secondary particles (called a shower) in the regolith all moving with almost the light velocity. This shower will have a net charge because of the electrons that are knocked out from the atoms of the regolith and thus emit radio-waves. Since the lunar regolith is very transparent to radio waves they may penetrate through the lunar surface and reach Earth where they may be detected with powerful radio telescopes. The first experiments using this approach were carried out with the Parkes telescope [Hankins+ (1996)], at Goldstone (GLUE) [Gorham+ (2004)], and with the Kalyazin Radio Telescope [Beresnyak+ (2005)]. In more recent years there has also a similar measurement been performed at a 
pathfinder of SKA, ATCA (the Australia Telescope Compact Array) with a 600 $\mathrm{MHz}$ bandwidth at 1.2-1.8 GHz [Bray+ (2015)].

The idea of Ger was to use the WSRT for this detection. It should be noted that the proposed WSRT observations differs from the usual astronomical observations. We are interested in detecting short, nanosecond scale, pulses emanating from the moon. This cannot be done with the standard equipment available at WSRT at the time however Ben Stappers had just developed his pulsar machine which was perfectly suited for storing the raw time-traces with zero integration time for later off-line processing.

When back at the KVI we thought more about the crazy idea of using the moon to detect neutrinos. The major differences with the previous GLUE experiment was the detection frequency $(200 \mathrm{MHz}$ instead of $2 \mathrm{GHz}$ ) and that we could use the WSRT as a phased array. Both turned out to give a major advantage over previous experiments.

Because of subtleties related to aspects such as the width of the Cherenkov radiation cone that is emitted by the particle shower in the regolith, internal reflection at the lunar surface, and the dependence of radio absorption in the regolith, the detection efficiency increases strongly with wavelength. In [Scholten+ (2006)] we could show that the detection efficiency for detecting UHE neutrinos via the moon scales with wavelength to the third power. This implies that one gains about a factor 1000 when shifting from $2 \mathrm{GeV}$ at GLUE to the $100-200 \mathrm{MHz}$ window at WSRT. This finding came as a great surprise to the UHE neutrino community.

By phasing the WSRT telescopes two rectangular beams can be formed where each covers one half of the moon. Since the main challenge in performing the observation is to distinguish true neutrino initiated pulses from noise much of this can be eliminated by requiring that a neutrino pulse should be seen in only one of the two beams. This condition eliminated much of the noise and increased our sensitivity. Another important suppression of noise is achieved by realizing that neutrino pulses from the moon have traversed the ionosphere and thus should be dispersed with the appropriate slanted total electron content (STEC) while noise pulses directly from sources on Earth should have no dispersion, or double this value when they reach WSRT through a Moon bounce.

In the off-line analysis these conditions can easily be implemented as well as additional ones such as the fact that lunar neutrino pulses should be bandwidth limited with an intrinsic duration of a few nanoseconds.

By the non-detection of a pulse of a certain magnitude (120 times the galactic background) over the duration of the observations (47.6 hours) we could set an upper limit on the flux of UHE neutrinos taking into account the attenuation of the neutrino pulse in the regolith as well as the geometrical acceptance. The re- sulting 90\% confidence flux limit [Scholten+ (2009), Buitink+ (2010)] is shown in figure 1 by the curve labeled WSRT. The band around the curve shows the possible error of the determined flux limit, mostly due to uncertainties in the attenuation of radio waves in the regolith.

The WSRT limits are compared with those of other competitive observations in figure 1. All these have used different techniques to cover a large area. The FORTE satellite [Lehtinen+ (2004)], observes the Greenland ice cap for radio flashes coming from neutrino interactions in the ice cap. ANITA is a balloon mission where the Antarctic ice cap is searched for radio flashes [Gorham+ (2009)]. Since for these observations the receiving antenna is much closer to the source than with our lunar

observations one is sensitive to neutrinos of lower energy. However with the Figure 1 The brown moon we have a target volume that is much larger than in either of these two experiments which means that at higher energies, since the flux is expected to drop with at least $\mathrm{E}^{-3}$, our experiment sets the most competitive limits. In the Figure also two model predictions are given. Waxman and Bahcall (1998) derived an estimate of the flux of neutrinos based on the observed flux of UHE cosmic rays assuming a generic model for neutrino production at the production sites of the cosmic rays. In Top-Down (TD) models UHE neutrinos are created from the decay of super massive relics $\left(M_{x}=10^{24} \mathrm{eV}\right.$ is used in the figure) formed at the time of the Big Bang. The data from our WSRT observation allows for setting limit on the flux of UHE neutrinos which is just touching the predicted flux of this TD model.

\section{The Future of NuMoon}

Over the past years, we began preparations to search for pulses of the impacts of cosmic particles in the Moon with LOFAR. Like the WSRT, the LOFAR HighBand Antennas provide an optimal frequency window for this technique. However, LOFAR has an increased sensitivity and the shape of the array allows for much more control over the beam shape, leading to better localization of pulses on the Moon.

Another improvement is the development of an online trigger. For the WSRT observations, the time-series data of all dishes was temporarily stored on a local computer at the observatory. Here, we had to run our data reduction software as fast as possible to make space for the next observations - typically within 24 hours or so. This was sometimes stressful, since the analysis needed up-to-date 
ionospheric electron density values as input, information that only became available the day after the observation.

The data rate of LOFAR makes it completely impossible to store hours of raw time-series data, so the whole detection pipeline has to work in real-time. This includes inversion of polyphase filters, forming 50 beams simultaneously to cover the lunar surface, correct for ionospheric dispersion, search for pulses, and make a trigger decision based on differences in the 50 beams. All of this has to be finished within 5 seconds - the length of transient buffer boards installed at each antenna.

The challenge is surprisingly similar to pulsar searches. The formation of multiple beams, the pulse searches, and the de-dispersion steps are the same, albeit on very different time-scales. It was therefore a logical step to seek collaboration with Jason Hessels who had just installed a GPU-based pulsar-search machine called DRAGNET. Using this cluster, we will be able to reach a sensitivity well below the previous WSRT limits.

The most interesting particles in this search may turn out to be cosmic rays instead of neutrinos. In contrast to neutrinos, cosmic rays convert all their power into the particle cascade that emits the radio pulse, lowering the energy threshold. This threshold comes very close to the cut-off energy observed by the largest cosmic ray detector arrays in the world: the Pierre Auger observatory and the Telescope Array.

The techniques that we are developing for LOFAR will in the future be applicable to the Square Kilometer Array. A first detection may be on the horizon and would be mind-boggling: detecting a single elementary particle impact at a distance of 380 thousand kilometers is surely the stuff only crazy astronomers dare to dream of.

\section{References}

M. C. Aartsen et al. [lceCube Collaboration], 2015, Phys. Rev. Lett. 115, 081102

A. Beresnyak, R. Dagkesamanskii, L. Zheleznykh, A. Kovalenko, and V. Oreshko, 2005. Astronomy

A. Beresnyak, R. Darts 49,127
Report

I.D. Bray et al. 2015, Phys. Rev. D91, 063002 .

S. Buitink et al. ,2010, Astron. Q Astroph. 521, A47.

R. Dagesamanskii and I. Zheleznyk, 1989, Sov. Phys. J.E.T.P. 50, 233.

P. Corham et al., 2004, Phys. Rev. Lett. 93, 4110

P. Gorham et al. (ANITA collaboration), 2010, Phys. Rev. D 82, 022004; erratum arXiv:1003.2961.

T. Hankins, R. Ekers, and J. OSullivan I.,1996, MNRAS 283, 1027.

IceCube collaboration, Science 12 Jul 2018:eaat2890 (DOI: 10.1126/science.aat2890)

H. Lethinen et al. ,2004, Phys. Rev. D 69, 013008.

R. Protheroe \& T. Stanev, 1996, Phys. Rev. Lett., 77, 3708.

O. Scholten et al.,2009, Phys. Rev, Lett. 103,191301

E. Waxman and I. N. Bahcall, 1998, Phys. Rev. D59, 023002.
Observations outside Radio Astronomy

\section{Chapter 17.2 Geodesy and Gravity at the WSRT}

Hans van der Marel and Roland Klees*

\begin{abstract}
strometry and geodesy are two complementary scientific disciplines that are closely related. In the past, optical observations both served positions of stars and the orientation of the Earth in space.
\end{abstract}

After 1957, with the launch of the first artificial satellites, geodesy became gradually less dependent on the stars. In the early 1960 s, the photogrammetric determination of the direction towards satellites equipped with flashlights, albeit still measured with respect to the stars as background, resulted in the formation of world-wide geodetic networks, as well as methods for the practical calculation of these networks, the computation of satellite orbits and the Earth gravity field. An enormous boost to the accuracy of the world-wide geodetic networks arrived with the development of highly accurate satellite laser-ranging systems to measure distances to specially designed geodetic satellites covered with retro-reflectors. These satellites, basically a heavy sphere to minimize drag and other perturbing forces, and covered with laser reflectors, played a crucial role in the determination of the Earth's gravity field. Satellite geodesy, as it was called, truly revolutionized geodesy.

In 1973, after the first successful experiments near Delft, the Delft University of Technology built the Kootwijk Observatory for Satellite Geodesy (KOSG) near Apeldoorn [2] . The observatory housed two satellite cameras for the photogrammetric satellite observation, satellite laser ranging (SLR) equipment, and the necessary workshops. The satellite laser ranging equipment was developed together with the Technische Physische Dienst in Delft (TPD). Large steps were made in the processing of the measurements towards the dynamical determination of all parameters involved: The Earth gravity field, accurate satellite orbits and station positions on the Earth. Sensing the Earth gravity field implicitly meant the determination of the center of mass of the Earth and motion of the Earth rotation pole in the same reference frame as the station positions. As the accuracy of the satellite ranging measurements improved, the next big 
challenge became the measurement of Earth deformation due to plate tectonics, Earth tides and various loading processes. Therefore, two transportable laser-ranging systems were developed (MTLRS-1/2), one for the German colleagues, and one for ourselves, which were deployed in the Mediterranean for observing the complicated plate tectonics in that area.

In the 1980's geodesy started to use differential phase measurements to Global Positioning System (GPS) satellites using specially developed geodetic receivers. First observations were done in dedicated campaigns, but the first continuously operating GPS receiver in the Netherlands was installed in 1991 at Kootwijk, which soon became one of the core stations of the International GNSS service (IGS) which was founded in 1994. With the development of transportable satellite laser-ranging systems and the upcoming geodetic applications of GPS (which eventually replaced the transportable satellite ranging systems), and because of diminishing funding, the department of geodesy had to stop the activities in Kootwijk and move the staff back to Delft.

After consultation with ASTRON, it was decided to continue the satellite geodetic observations in a newly established Astrometric Geodetic Observatory at Westerbork (AGOW) [3]. The decision to move the observations to Westerbork was made easy by the fact that TUD and ASTRON already cooperated in geodetic Very Long Baseline Interferometry (VLBI) campaigns. The co-location of several important geodetic observation techniques at a single site was seen as a great opportunity.

The geodetic part of the AGOW observatory was built in 1996 and consisted of:

- Continuously operating GPS (GNSS) receivers as part of the International GNSS Service (IGS) and national Active GPS Reference System (AGRS)

- A very stable $24 \mathrm{~m}$ high steel mast, on a concrete foundation, for the GPS observations.

- Underground concrete block of $3 \times 3 \times 3 \mathrm{~m}^{3}$ in a climate controlled bunker for absolute and relative gravity measurements.

- Universal 9x9 $\mathrm{m}^{2}$ concrete platform for transportable VLBI and SLR systems.

- An underground NAP (Normaal Amsterdams Peil) marker, connected by primary levelling measurements to other underground markers.

In 1997 the Netherlands Commission for Geodesy (NCG) formed a task group to investigate the added value of the synthesis of different measurement techniques at Westerbork [3]. Central in its report [3] is the role of the AGOW as a global anchor point for the Netherlands, especially in relation to the anticipated sea-level rise and challenges faced in the global determination of the height component. According to the task group, the focus should be on long continuous time series of parallel geodetic measurements, in particular for the height component, in combination with international efforts on the establishment of geodetic reference systems. The task group also recommended the feasibility of the participation of one of the telescopes in regular geodetic VLBI campaigns.

The AGOW was officially opened on 29 September 1999 with a symposium in Dwingeloo and visit to the observatory at Westerbork. The presentations of this symposium were published [1].

A rigid and stable GPS antenna mast is necessary in order to have a clear unobstructed view of the GPS satellites because of the tree growth at Westerbork. See Figure 1.

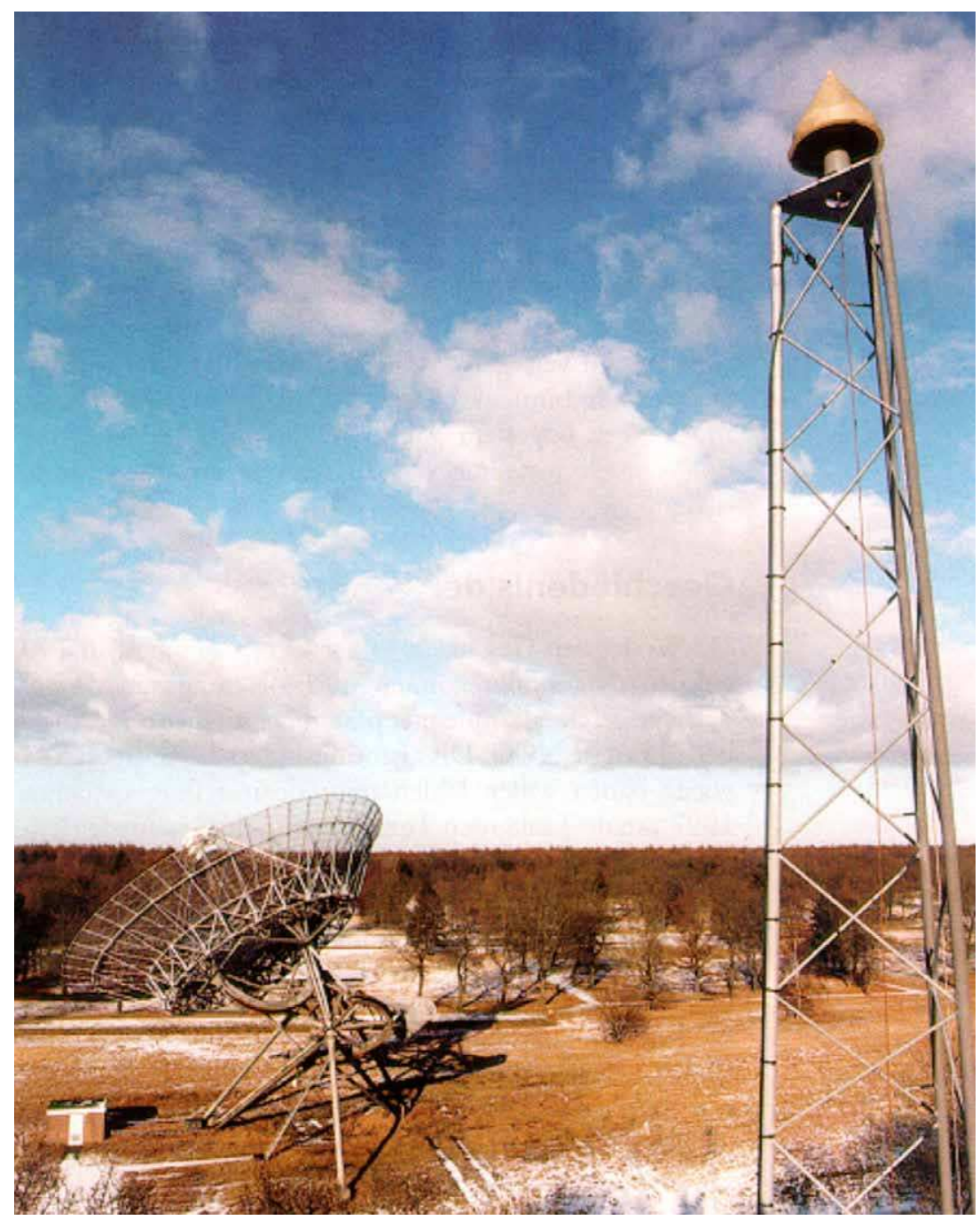


The mast has a special invar-wire construction to keep the influence of temperature changes on the vertical position of the antenna to an absolute minimum, while it will not move by more than $2 \mathrm{~mm}$ in a gale force storm. The position of the antenna is checked once per year using a local survey and levelling to the nearby underground NAP marker, thereby connecting the GPS heights directly to NAP.

The two GPS receivers are placed in the cellar of the main building. Both receivers are connected to the hydrogen maser at Westerbork, providing a very stable reference frequency for the GPS. Over the years the receiver hardware has changed and receivers have been upgraded to full GNSS (Global Navigation Satellite Systems) capable receivers supporting not only GPS, but also the Russian built Glonass system, the European Galileo and Chinese Beidou systems. However, the same GPS antenna has been in use since 1997, making this station a very unique and stable reference point for the International GNSS service (IGS) and International Terrestrial Reference Frame (ITRF). For the maintenance of the ITRF and anchor points in the Netherlands it is important to keep GPS antenna changes to an absolute minimum, as installing a new antenna will inevitably results in a small discontinuity in the time series, especially in the height. Westerbork is therefore unique and important point in the ITRF reference frame because of the long term stability and long uninterrupted time series, see Figure 2, in particular in combination with Kootwijk for which there is a long overlap and history goes back to 1991.

\section{WSRT}

Cumulated residuals
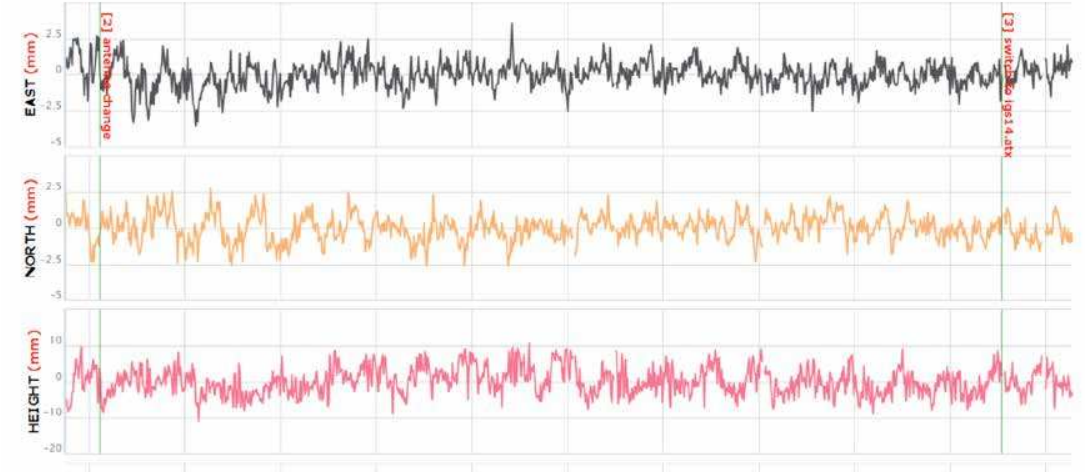

GPS observations from Westerbork are used for the determination of accurate satellite orbits for almost all geodetic and Earth observation satellites, which are in turn used for computing atmospheric parameters such as the integrated water vapour content and total electron content, and many other applications.
Measurements of absolute gravity at Westerbork have been done since the bunker became available at the end of the twentieth century. They are part of a long-term plan to monitor the vertical stability of the Dutch NAP height network. The observed changes in absolute gravity are transformed into vertical displacement rates. Linking the Westerbork station to the NAP network using spirit levelling, measured relative vertical movements of NAP height markers can be transformed into absolute movements. The information about absolute vertical movements of NAP height markers is used determine absolute vertical land motion at the tide gauge stations along the Dutch coast. In this way, variations in sea level caused by changes in the volume of water in the oceans can be extracted from the tide gauge records, a prerequisite for a proper interpretation of observed relative sea level variations.

Before 2006 , the measurements were done by the University of Luxembourg, because no absolute gravimeter was available in the Netherlands. This changed in 2006 , and since then, TU Delft measures absolute gravity once a year in Westerbork and other stations in the Netherlands (currently, these stations are located in Epen, Kootwijk, Zundert, Oudemirdum, and Oudeschild). Figure 3 shows the observational record of absolute gravity variations after corrections for instrumental errors and geophysical signals, including tides and ground water table variations.

The linear trend in gravity is estimated as $-0.034 \pm 0.041 \mathrm{microGal} / \mathrm{yr}(1 \mathrm{micro}-$ $\mathrm{Gal}=10^{-8} \mathrm{~m} / \mathrm{s}^{2}$ ). This corresponds to a vertical motion rate of $0.17 \pm 0.21 \mathrm{~mm} /$ yr over the last 20 years.

Not all of the original ambitions of 1997 have been fulfilled. For instance geodetic VLBI, although done periodically, and satellite laser ranging (SLR), have been completely replaced by GNSS observations. However, the Astrometric Geodetic Observatory at Westerbork (AGOW) has become one of the core geodetic reference stations in the world and is crucial for geodesy in the Netherlands. Its observations are used by countless groups in the world for reference frame maintenance, computation of satellite orbits, monitoring of subsidence and sea level change, and atmospheric research. WSRT is an acronym that is immediately recognised by geodesists around the world, although not all geodesists will immediately associate WSRT with radio telescopes.
Figure 3 Time series of absolute gravity measurements at The estimated trend is $-0.034 \pm 0.04$ microGal/year $\left.10^{-8} \mathrm{~m} / \mathrm{s}^{2}\right)$ 
[1] Aardoom, I. (ed), Samen meten, beter weten. Voordrachten gehouden ter gelegenheid van de Nederlandse Commissie voor Ceodesie 38, Delft, 2000, 49 pagina's, ISBN 243 : 978 go 67322726 ISBN-10: 9061322723 . https://wwinceo nl/index php?option=com k2\&view=item\&id=2357: 38---aardoom-samen-meten-beter-weten\&ltemid $=178 \&$ lang $=n$

[2] Poelstra, T.J., A new satellite observatory at Kootwijk, Nederlandse Commissie voor Ceodesie 17, Delft, 1974. 26 pagina's. ISBN-13: 978906132024 1. ISBN-10: 9061320240. htps. / www.ncgeo.nl/index.php?option=com_k2\&view=item \&ld=2335:-5s-17-t-t-poelstra-a-new-satellite-observatory-at-kootwijk\&ltemid=178\&lang=n|

[3] De wetenschappeli)ke rol van het astionetrisch-geodetisch observatorium Westerbork (The scientific role of the astrometric-geodetic observatory Westerbork), Uitkomsten van een verkenning door Geodesie. Internal report.
Observations outside Radio Astronomy

\section{Chapter 17.3 lonosphere, the WSRT, LOFAR and Space Weather}

Arnold van Ardenne, Maaijke Mevius*

\begin{abstract}
round 1980 Titus Spoelstra was responsible for the Westerbork data reduction. Because of this responsibility, his scientific interest and coninuing quest for improving the precision of the data Titus wished to understand better the effects of the ionosphere on the data set. His ultimate goal was, of course, to seek ways to correct the data using this knowledge.
\end{abstract}

As ASTRON was not tasked to conduct ionospheric research, of which effects and insights were considered merely byproducts, Titus contacted in the early eighties Hennie Kelder from the Department of Geophysics at KNMI the Royal Netherlands Meteorological Institute. This contact resulted in a joint paper in the subject "Effects of the Ionosphere on Radio Astronomy" which appeared in June 1984 (Radio Science, Volume 19, Number 3, Pages 779-788) spelling out the refraction effects of the ionosphere on the WSRT fringes as observables.

Titus approach was that a better understanding was necessary to apply radio astronomical data corrections for both local WSRT interferometric work as well as for VLBI. While tracking the radio source ${ }_{3} \mathrm{C}_{2} 86$ at $608,5 \mathrm{MHz}$ (the nominal $50 \mathrm{~cm}$ wavelength) Titus and Hennie measured the solar-driven, notably diurnal, phase effect (amplitude and polarization angle, the latter from KNMI data) in the ionosphere. This effect of the sun on the ionosphere is clearly visible in the top picture on the left (copied from the article) from which it was noted that the phase effects are most notable during sunrise and sunset. But there are also many irregularities caused by "medium" scale Travelling Ionospheric Disturbances (“TID"'s). Further investigations in 1982, with the WSRT, used satellite passages in the eastern and western skies as artificial radio sources. The measured phase behaviour of a satellite signal is shown in the lower figure (from the same article). These studies improved the knowledge of TID's (scale size, direction of propagation, time scales) and their effects on radio interferometry As a general main conclusion the article therefore mentions that "Radio interferometers turn out to be excellent instruments to 
Figure 1: The two pictures are taken from the 1984 article by Hennie Kelder and Titus Spoelstra showing the effect of the sun on the measured Westerbork phase data and of Travelling lonospheric Disturbances ("TID"'s) using satellites as artificial radio sources (see the text).

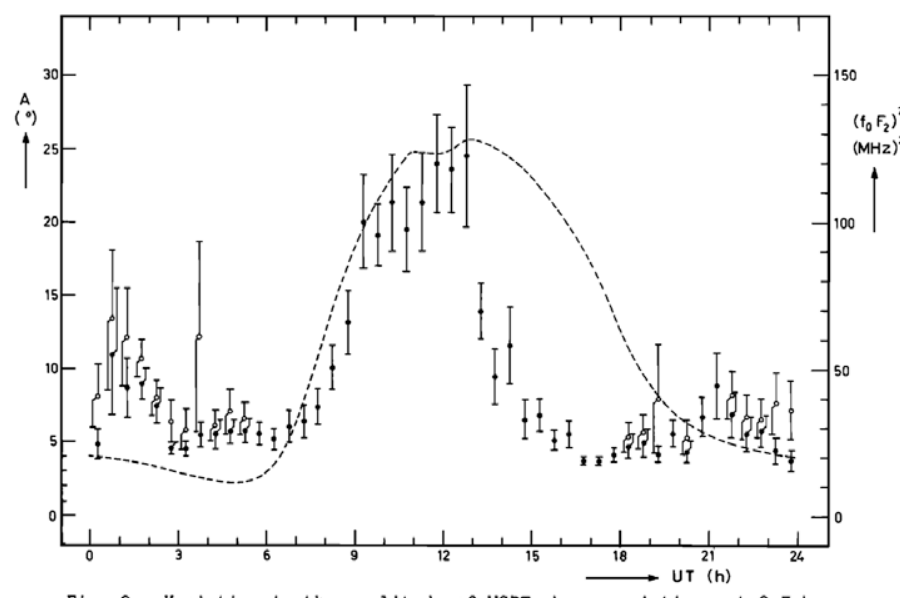

Fig. 2. Variation in the amplitude of wSRT phase variations at $2.7 \mathrm{~km}$
baseline due to ionospheric irregularities as a function of time. Solid circles indicate that data with apparent periods less than 4 min have no 1ine represents the average $\left(f_{\mathrm{F}_{2}}\right)^{2}$ variation with time. Local noon is at
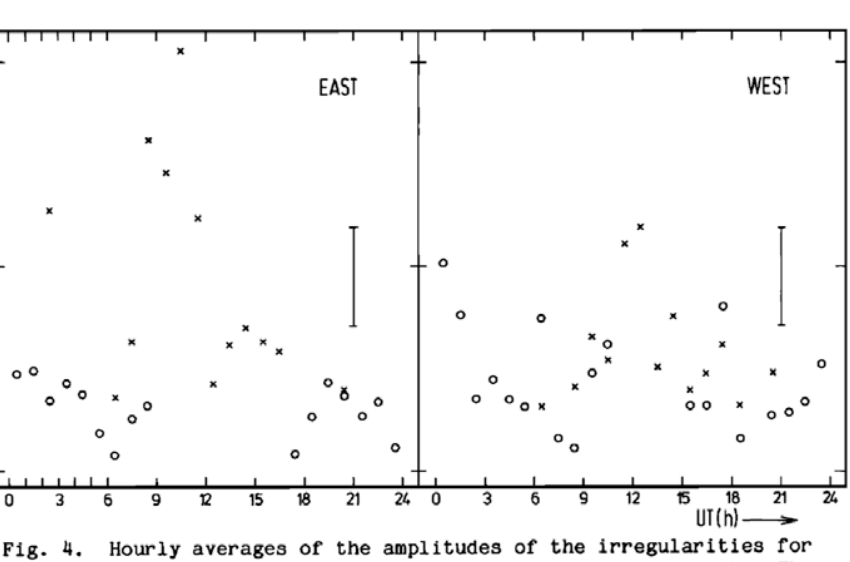

Fig. 4. Hourly averages of the amplitudes of the irregularities for
satelilite passages in the eastern as well as in the western sky. The different behavior for waves with apparent periods of $4.6 \mathrm{~s}$ or less
(circles) and medium-scale TID's (crosses) is indicated. The error ba (circles) and medium-scale TID's (crosses)
indicates the spread per averaged data point.

“The absence of medium-scale TID's during the night suggests that the sun may be the main "engine"'.

Critically proofread by Richard Strom, it proved the beginning of more thorough work in particular on TID's. This is reflected in the article by Hennie Kelder and Titus Spoelstra with the title Medium scale TlDs observed by radio interferometry and differential Doppler techniques (Journal of Atmospheric Terrestrial Physics, Vol. 49, No. I, pp. 7-17, 1987). It again demonstrated the importance of (lower) frequency radio interferometry techniques to understand the ionosphere and in this case postulating a hypothesis about the cause of TID's.
In a relatively unknown but nice overview in 1997 "The Ionosphere and Radio Interferometry (Annali di Geofisica, Vol.XL, nr4, pp 865-914, August 1997)" the previous work was summarized and expanded. In the Netherlands through the PhD thesis work of Peter van Veldhoven (now senior researcher at KNMI), additional work at the lower WSRT frequency of $327 \mathrm{MHz}$ and through comparisons of results from other telescopes and GPS data. Important additiona insights dealt with the effect of ionospheric scintillation and instrumental effects. After these studies there is no doubt that interferometric radio astronomy and ionospheric research go hand in hand.

Titus retired in 2008 (see also the AJDI archive and then look for the one of o7 11-2008) and his ailing health led to his premature death on 30 April 2010. The connection between radio interferometry and the ionosphere, that was scientifically explored by Titus, received less attention after his demise but is becoming again a very relevant subject.

The postulated conclusion of 1984 that the sun is the main engine for effects in the ionosphere by now is taken for granted. In fact, the magnetic activity of the sun affects the magnetosphere and ionosphere of the Earth and can have pronounced effects on modern technology (commonly called the effects of space weather). A good understanding of the heliosphere and the ionosphere are paramount to now cast or forecast these effects. As it happened, the LOFAR telescope is evolving into a formidable instrument for this purpose.

With the LOFAR telescope we gain insight on the ionosphere in various ways. Since ionospheric diffractive delays are a main source of calibration errors, calibration parameters give a direct measure of the differential integrated electron content over the array. Amplitude scintillation can be measured with single station data while moving scintillation patterns are observed if the data of more stations is combined. Also, dual polarization elements allow the measurement of rotation of the polarization angle of a linear polarized signal due to the interaction with the ionospheric plasma and the Earth magnetic field, known as Faraday rotation. Interestingly, even an unpolarized signal can become artificially polarized if the Faraday rotation effect above the two arms of an interferometer differ.

We have used the LOFAR station beam data to find ionospheric scintillation patterns of a bright astronomical source, such as Cas A. Comparing the scintillation amplitudes of several stations, one gets a direct view of the patterns in the ionosphere at the station positions projected along the line of sight. Imag ing these patterns in time this gives a movie of the ultra-fine structures in the ionosphere, moving around above the LOFAR core. Although at mid-latitudes, at these frequencies amplitude scintillation is observed almost continuously, contrary to what has been observed with GNSS measurements at higher frequencies (R. A. Fallows et al, 2016 ApJL 828 L7). 
In interferometric mode, the data of all stations are correlated and averaged to typical 1 second time resolution. Since, in this mode the system is only sensitive to the phase difference of a signal arriving at two stations, the measured ionospheric effects are also mainly differential. A linear gradient in the ionospheric total column electron content (TEC) over the array will cause a (frequency dependent) shift of the measured position of a source. Higher order terms will cause source deformations in the image plane. Typically, the ionospheric variation in a single direction can be described by a linear gradient for the LOFAR core, where higher order terms show up at longer baselines. When imaging the position shifts of a large number of sources inside the LOFAR beam as a vector field, larger scale disturbances, like TIDs or duct like structures, become visible over an area corresponding to the LOFAR beam. Although a single pointing of the LOFAR HBA beam only corresponds to about 10 degrees, and therefore to about 50 square $\mathrm{km}$ at an altitude of $300 \mathrm{~km}$, it is possible to use LOFAR in simultaneous multi beaming mode, sacrificing bandwidth for more pointings.

Figure 2: 2 minutes shapshot of position
shifts of $\sim 800$ sources of combined data of 7 LOFAR beams. The black lines show the projected magnetic field lines. magnetic field lines. structure function of the same obser. vation, binned for angle wrst magnetic field (colorbar). The fleld (colorbar). The along the magnetic feld lines are clearly

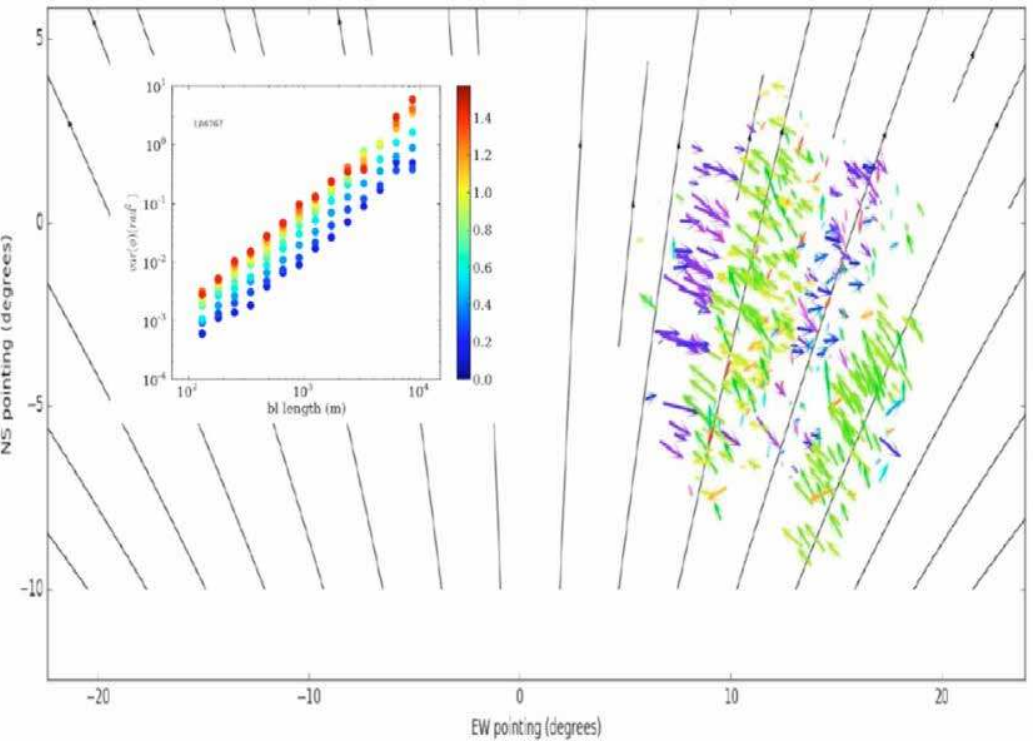

By comparing a model of the sources in the sky to the actual data during calibration, station based phase errors are estimated. By making use of the wide bandwidth and the typical frequency behavior of ionospheric delay, the ionospheric effects are separated from other (instrumental) phase effects. To first order the ionospheric phase errors scale with the inverse of frequency $(1 / \mathrm{f})$, although at the lowest LOFAR frequencies $(<40 \mathrm{MHz})$ third order frequency effects (scaling as $1 / \mathfrak{f}^{3}$ ) become visible. As LOFAR can measure phase errors with very high accuracy, differential integrated total electron content ("TEC") can be measured with an accuracy smaller than 1 milli-TECU $\left(10^{13}\right.$ electrons/ $\mathrm{m}^{2}$ ), using a typical HBA calibrator observation and 10 sec integration. These are "local" if the phase solutions are used in the direction of a single calibrator when the differential TEC on an area in the ionosphere equal to the footprint of LOFAR is measured (Mevius et. al., 2016, Radio Sci. 51, 927-941)

Faraday rotation as the second order phase delay effect, scales with $\left(1 / f^{2}\right)$, causing a phase delay of circular polarized signals like those from GNSS. With LOFAR it becomes visible as a rotation of the linear polarization angle. Given an Earth magnetic field model, the measured time varying rotation angle of a polarized source can give a direct measure of the absolute TEC (SotomayorBeltran C. et al 2013 A\&A 552 A58). Even for an unpolarized source the effect is visible if the ionospheric Faraday rotation angle above the stations of a baseline differ, either because of differential TEC, or because of a slightly different parallel magnetic field vector. See: de Gasperin et al (2018, AaA, https://doi. org/10.1051/0004-6361/201833012) showing all three orders of ionospheric phase effects in LBA calibrator data.
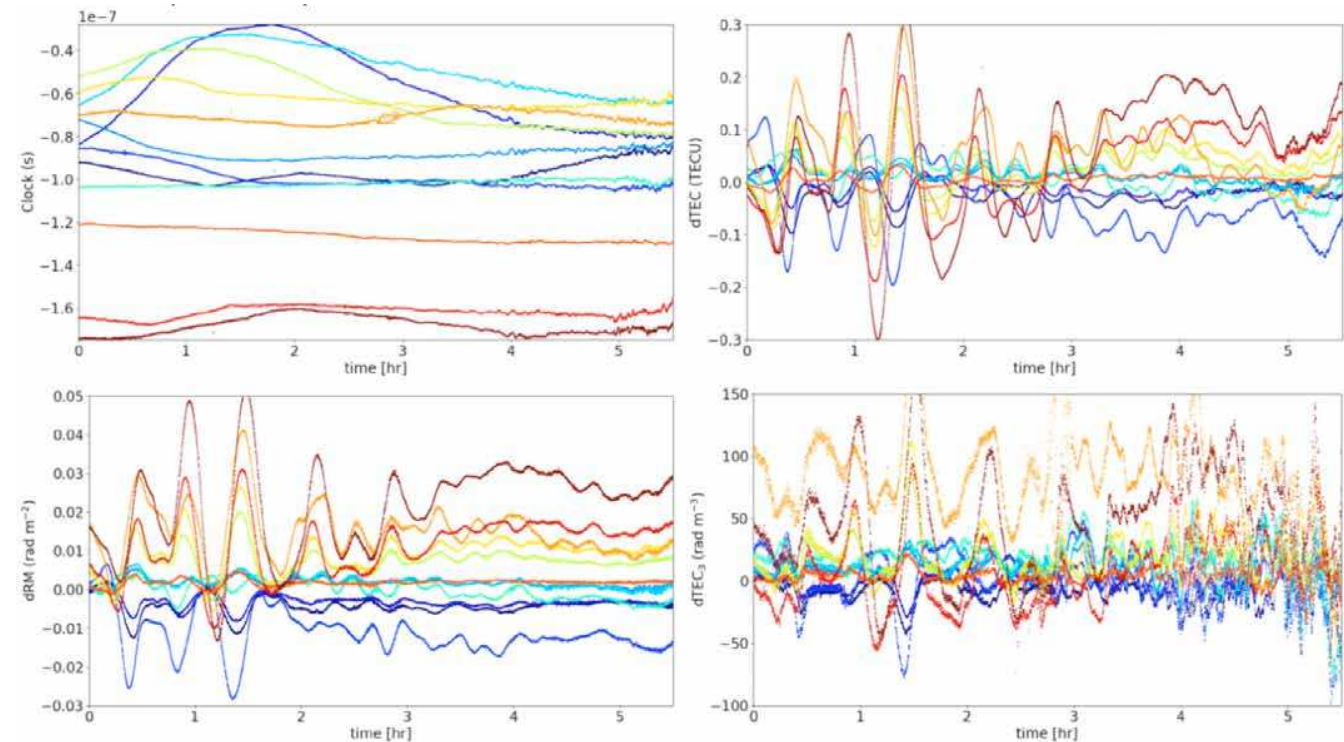

Figure 3: from de Gasperin et al.. From top-left to bottom-right: Instrumental clock delay (in s). Total electron content variation along the observation (TECU). Faraday rotation (in rad $\mathrm{m}-2$ ). Ionospheric third-order effect (in rad $\mathrm{m}-3$ ). All values are differential between CS 001 (assumed constant at o) and all remote stations (from blue to red in alphabetical order).

This work and other observing capabilities of LOFAR made it possible to now get an approved role (e.g. through an ESA contract) in exploring its use for Space Weather for both civil and defense purposes but in any case for societal benefit. It is interesting to note that the foundations were laid by people like Titus Spoelstra an Hennie Kelder more than thirty years ago. 


\section{The search for NASA's Mars Polar Lander}

Early December 1999, at more than The Mars Polar Lander

Early Decention 300 millon klometres from Earth, spacecraft from NASA landed on the planet Mars: the Mars Polar Lander (MPL). The main goal of the MPL was to find traces of water in the bottom of Mars. Unfortunately, nothing has been heard from the ander since the start of the decent towards the red planet. What happened? Did it crash, was the communication broken, or something else? Questions that might be answered by observing possible radio signals from the MPL, using the Westerbork Synthesis Radio Telecope. On Wednesday, January 26, 2000, it all started for us: we received an email from NASA asking if we would like to contribute to an experiment to determine whether the Mars Polar Lander was still 'alive'. A very interesting journey started...

On January 3, 1999, the American space organization NASA launched the Mars Polar Lander. On December 3, 1999 the decent started to the surface of the red planet. The communication between the MPL and the Earth took place via an X-band system. But this system did not work! A second option was an UHF link On the MPL a UHF transceiver was mounted, with which a connection could be made with a satellite that orbits around Mars. This satellite, the Mars Clobal Surveyor (MCS) takes pictures of the planet at an altitude of $380 \mathrm{~km}$. The MCS can serve as a relay station between the MPL and the Earth. But this function was also not working.

The final option was trying to pick up the UHF signal directly from the MPL! This signal is very weak: a

Figure 1: Artist impression of the Mars (C) JPL/NASA)

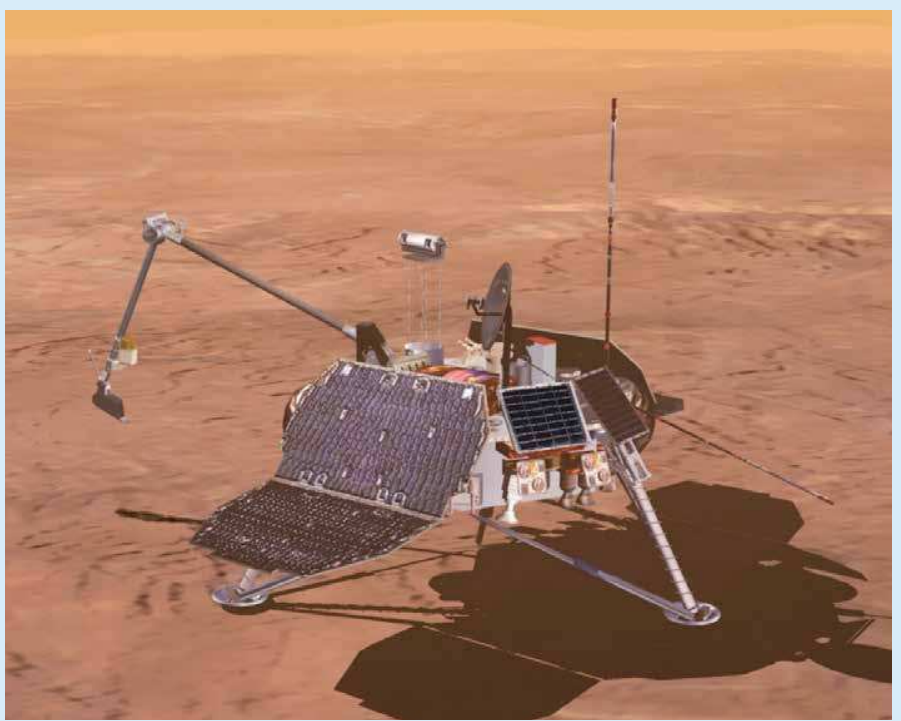

transmitter of about 10 Watt at a distance of 300 million kilometres from Earth! Due to the weakness of this signal, a radio telescope must be used to even have a chance to detect the signal. The radio telescope in Stanford (California, USA) reports that it might have seen the signal in a measurement of January 4. The detection is doubtful, but nonetheless encouraging to look further.

There are only three radio telescopes in the world that are sensitive enough to detect the signal at the frequency emitted by the MPL: the frequency emitted by he MPL: the Stanford telescope in Californ (USA), the radio telescope in Jodre Bank (England) and the Westerbork Synthesis Radio Telescope in the Netherlands, of which the WSRT is by far the most sensitive on

What are we looking for?

The UHF communication between the MPL and the MCS takes place via an FSK modulated signal at $401.5 \mathrm{MHz}$. This means that at a distance of $+/-128 \mathrm{kHz}$ around the central frequency of $401.5 \mathrm{MHz}$ a signal should be found. But this frequency is somewh by the fact that Mars moves with respect to the Earth. Mars moved away from Earth during the me surements at a speed of almost $10 \mathrm{~km} / \mathrm{s}$, taking into account the rotational speed of both Mars and the Earth. As a result, the original frequencies are reduced by about $10 \mathrm{kHz}$.
The experiment

It was agreed with NASA that they would program the MPL in such a way that the UHF transceiver on the MPL would be turned on at exactly 15:30 (UT). This will last for half an hour. Then the electronics must cool down for two hours, which the whole thing would be repeated once more. The distance between Mars and the Earth was about 300 million kilometres, whic means that the signal takes 16 minutes and 55 seconds to reach Earth.

\section{The real challenge}

The real challenge was the fact that the signal was very very weak. We use a special mode at the WSRT, in which we add up all the 14 telescope signals to capture the signal. The difference between the signa from the MPL and the strongest

signal in the band, the TV-smilde Nederland-2 signal, is almost $200 \mathrm{~dB}$ (that is a factor of about 100.000.000.000.000.000.000). Using the PuMa (Pulsar Machine) it might be possible to detect the MPL signal. The PuMa is an in

strument built by the University of Utrecht in a collaborative pritect Utrecht in a collaborative project between ASTRON and the univer sities of Utrecht and Amsterdam. To make this possible, we had to eliminate all possible interference around the MPL frequency (401.5 $\mathrm{MHz}$ ). So, we completely closed the park in Westerbork (nobody was allowed to come in), we shut down all the electronics not needed in the observations (computers, telephone equipment, alarm). Local HAM radio amateurs were asked not to transmit during the observations. And even the KNMI was asked to arrange that weather balloons in

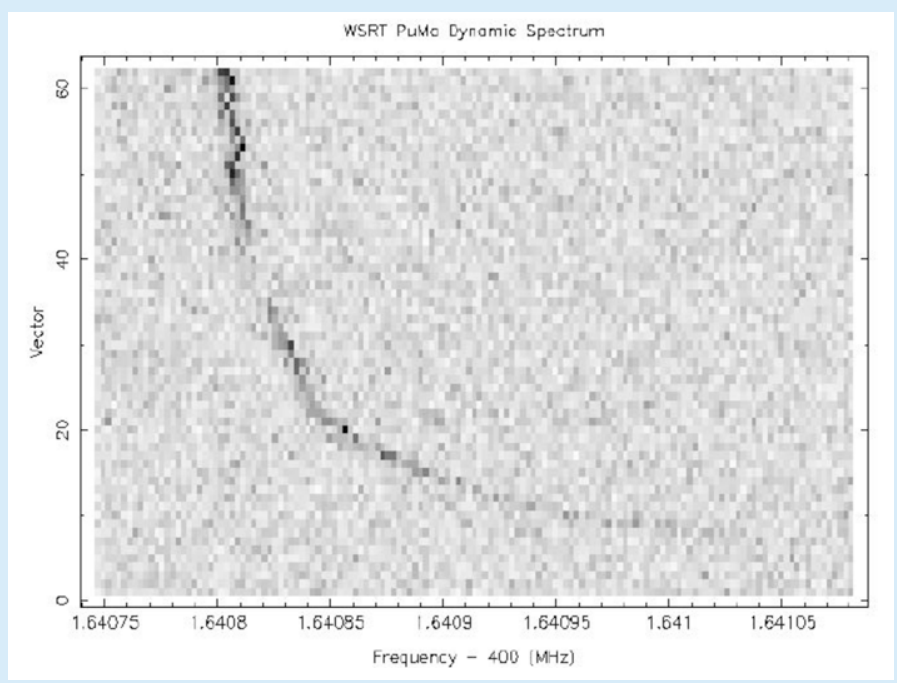

Figure 2: The first possible signal from the MPL. 
Figure 3: The complete time series of frequency scale is not correct). the possible MPL signal. (note that the

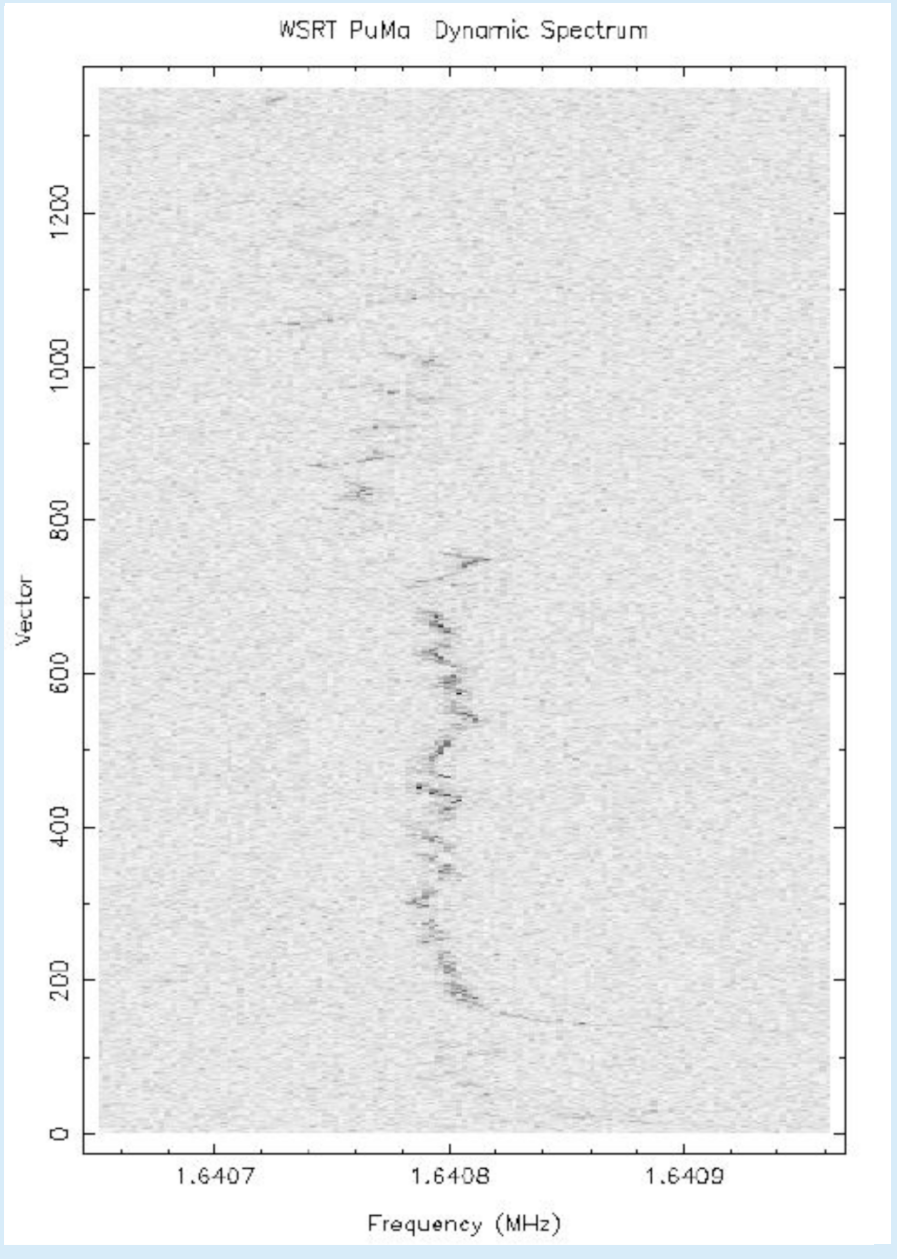

After the first few minutes were observed, the data was processed. After an hour of processing the firs picture appeared on the screen. And to our big surprise, there was actually a signal on the screen (see Figure 2). Did we really detect the $\mathrm{MPL}$ ?

\section{The big day.}

Friday, February 4 (2000) was the big day. After the system had been perfectly tuned and all RFI had

been removed as well, it was time to make the first MPL detection at exactly 15:46:55 UT. Except for a very tense atmosphere at the observatory, nothing happened. First the recorded data had to be processed.
And the frequency was exactly what was expected as well.

Logically, the mood was euphoric at that moment. Everyone was really signal! But .... of course, we remain more characteristics of a possible MPL signal: a second carrier of the FSK signal must be there as well, and if we performed an on-off se. quence the signal must disappear. So, we really had to check these facts before we could champagne bottle. signal! But ... of course, we rem scientists and therefore: check and check again. After all, there were course not, so we redid the evable. The signal started exactly on the right time (within a second). The signature of the signal was exactly what we expected: a drift in the first seconds and stable afterwards. quence the signal must disappear.
After a night of number crunching, finally in the morning the complete picture showed up as can be seen in Figure 3.

After seeing this image, we wer quite disappointed. The second signal for the FSK modulation wasn't there, but more important, the

signal didn't disappear in our on-off source (Mars) actions. Therefore, it couldn't be from Mars. Unfortu- nals in the UHF band. A couple of months later, NASA indicated that there were a number of prob with the MPL and that it was very likely that it actually had crashed on the Martian surface. This was confirmed by NASA a few years later. Nevertheless, the entire experiment for the WSRT was very successful and absolutely fun to do.

\section{Nothing found - Experiment}

successful

Therefore, our conclusion was that the MPL no longer broadcast sig.
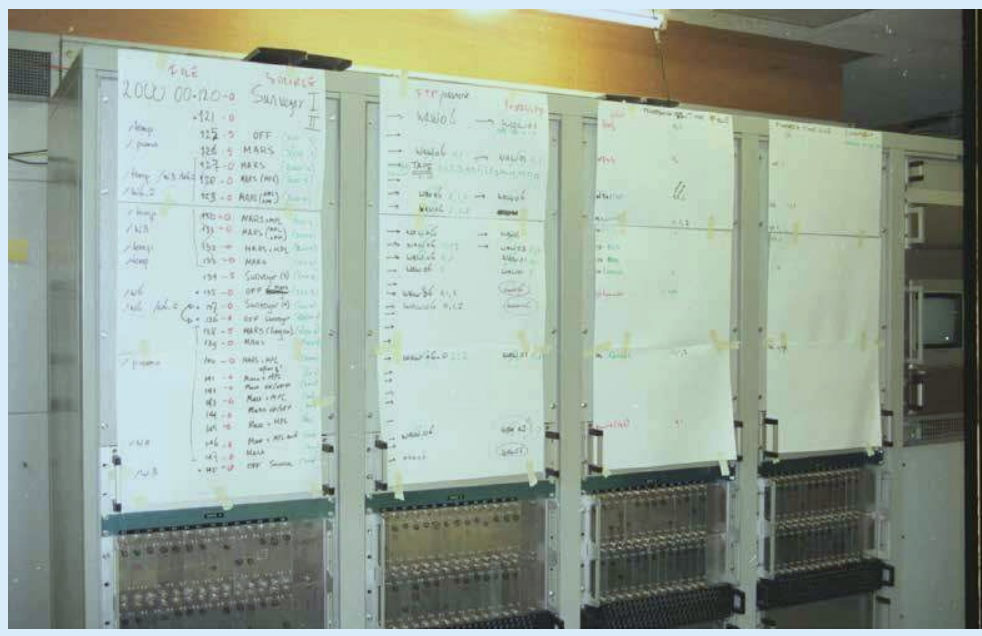

Figure 4: the schedule of the
Figure 5: Ben Stappers, Peter Fridman and Marco Kouwenhoven working on th PuMa data in the evening of 4 Februari 2000. Not sure!?

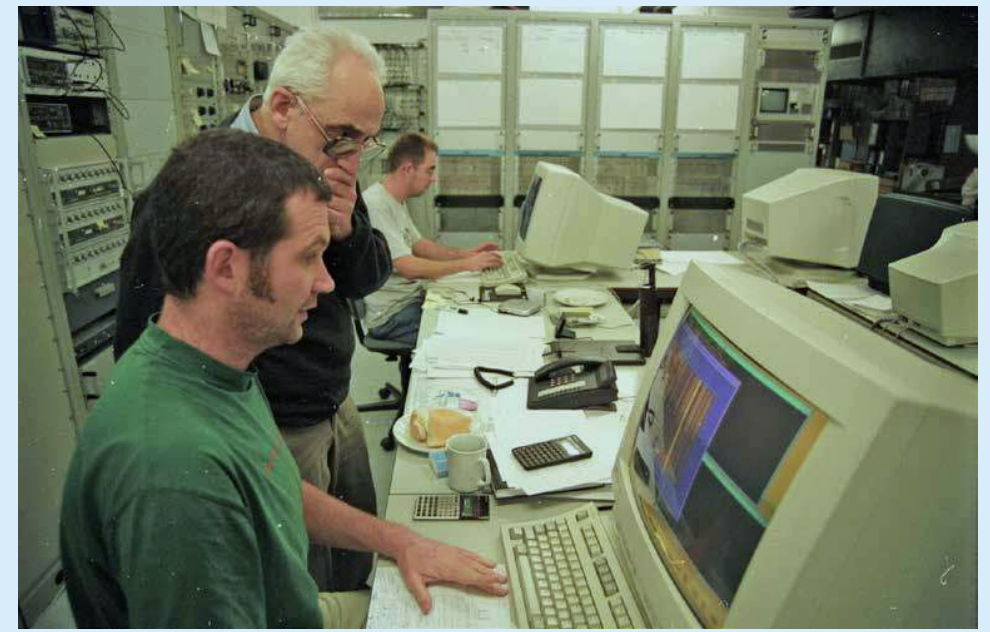




\section{Observations outside Radio Astronomy}

\section{Chapter 17.4 A Westerbork Radio Telescope for Galileo Monitoring}

\author{
Arnold van Ardenne, Hans van der Marel, Koos Kegel, Andre Bos*
}

\section{Introduction}

In the first decade of this millennium, Europe started to build its own Global Navigation Satellite Service ("GNSS") system called Galileo. Aimed to be ready in a few years form now, it will consist of 28 satellites of which 22 have been successfully launched so far.

Leading up to this, preparations started in 2007 to suitably modify one telescope out of the 14 of the Westerbork Synthesis Radio Telescope ("WSRT") array in order to support the early assessment of the Galileo system in the first, In Orbit Validation phase. While preparations continued, a contract was awarded through the European Space Agency in the end of 2008 to develop a Signal In Space monitoring system for observations on the early Galileo test satellites Giove A and B (see: http://www.esa.int/esapub/br/br251/br251.pdf) and the first 4 flight models of the Galileo satellites.

This Signal Monitoring Facility ("SMF") system was initially using telescope 6 with a suitably modified multifrequency frontend receiver to allow for the required calibration and circular polarization observations for GNSS usage. The MFFE build under the leadership of Gie Han Tan, now at ESO, has been described elsewhere in this book.

As very stringent requirements called on the accurate calibration of group-delay and absolute power levels, the telescope apex was equipped with a suitable comb generator. Furthermore, a new "software" receiver allowed for the SIS performance analysis. This resulted in the successful qualification by Thales Alenia Space(It.) under auspices of the European Space Agency (ESA) and its

* This contribution is modified from the published paper "A Westerbork Telescope for GNSS Signal In Space Monitoring" for the AT-RASC URSI conference in Gran Canaria, 2018. Andre Bos, from Science \& Technology B.V., Delft, Neth., has been collaborating since the early GNSS activities in ASTRON.
Technical Centre (ESTEC) in early 2014 at the onset of further Galileo satellites in the next phase.

The picture shows the team in front of the then GNSS telescope RT6. ASTRON collaborators from the Technical University Delft and from TNO were not present but contributed as well. The authors are (from left to right) Arnold van Ardenne (third form left), Koos Kegel and Andre Bos (second from left) and Hans van der Marel.

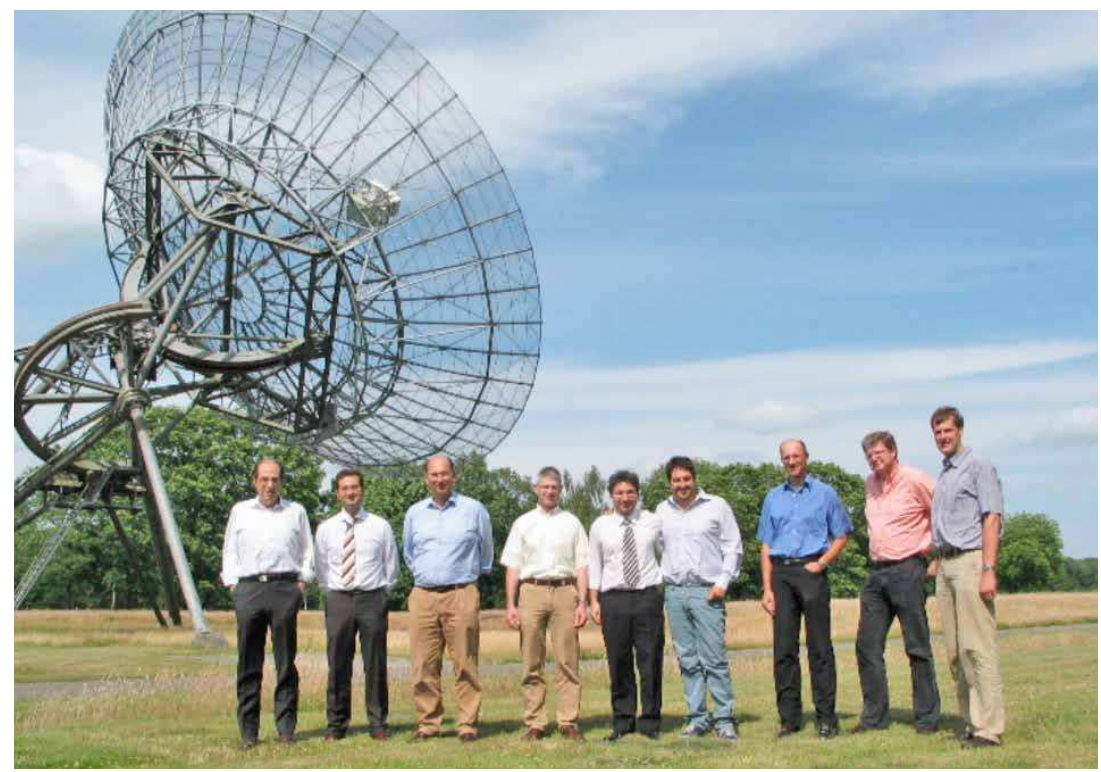

Through this success, further developments lead to a new prototype wideband receiver and an awarded contract with the European GNSS Agency ("GSA"). This facilitated the move of the facility to another telescope ("o") of the WSRT used for limited VLBI programs and limited other radio astronomy purposes.

\section{System Layout}

The principle system layout is given in Figure 1 below depicting the relation between the SIS monitoring and analysis receiver which functionality is largely software PC-based.

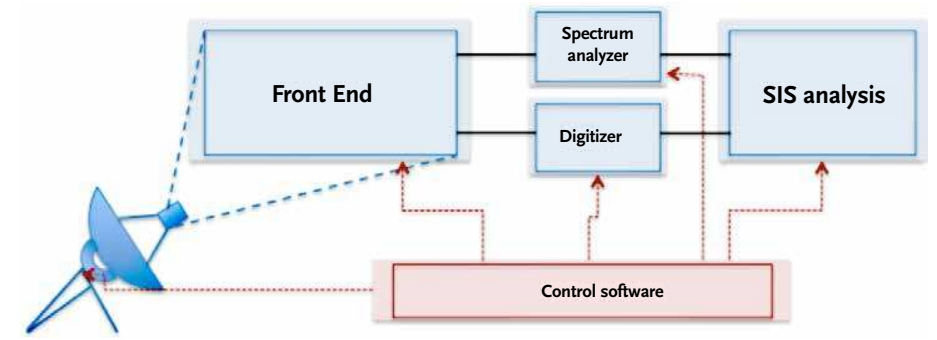

Figure 1. Principle system block diagrams. The default High wain Antenna is Telescope o, duplicate cooled receiver also a modified MFFE frontend, allows for redundancy. 
Table 1 summarizes pertinent data of the telescope used for the GNSS observations using a modified cryogenically cooled receiver i.e a modified MFFE. The telescope includes a comb generator in the apex for accurate group-delay calibration an approach derived from geodetic VLBI.

Table 1. Pertinent data describing the High Gain Antenna.

\begin{tabular}{ll}
\hline Antenna type & $\begin{array}{l}\text { 25m High gain antenna, equatorial mount, } \\
\text { prime focus }\end{array}$ \\
\hline Antenna gain & $48 \mathrm{dBi} @ 1.2 \mathrm{CHz}$ \\
Polarization & $\mathrm{RHCP} / \mathrm{LHCP}$ or X/Y \\
Frequency range & $1.12-1.65 \mathrm{GHz}$ \\
\hline Instantaneous bandwidth & $160 \mathrm{MHz}$ \\
\hline Half-beam width & $0.5 \mathrm{deg}$. \\
\hline Pointing accuracy & $0.005 \mathrm{deg}$. \\
\hline Slew rate & $18 \mathrm{deg} . / \mathrm{min}$ \\
\hline System temperature & $25 \mathrm{~K}$ \\
\hline Calibration & Apex comb generator \\
\hline
\end{tabular}

Figure 2 presents the full SMF system in more detail depicting the left-/righthand circular outputs from the HGA. Other instruments are for RF monitoring and data analysis, storage and -user distribution.

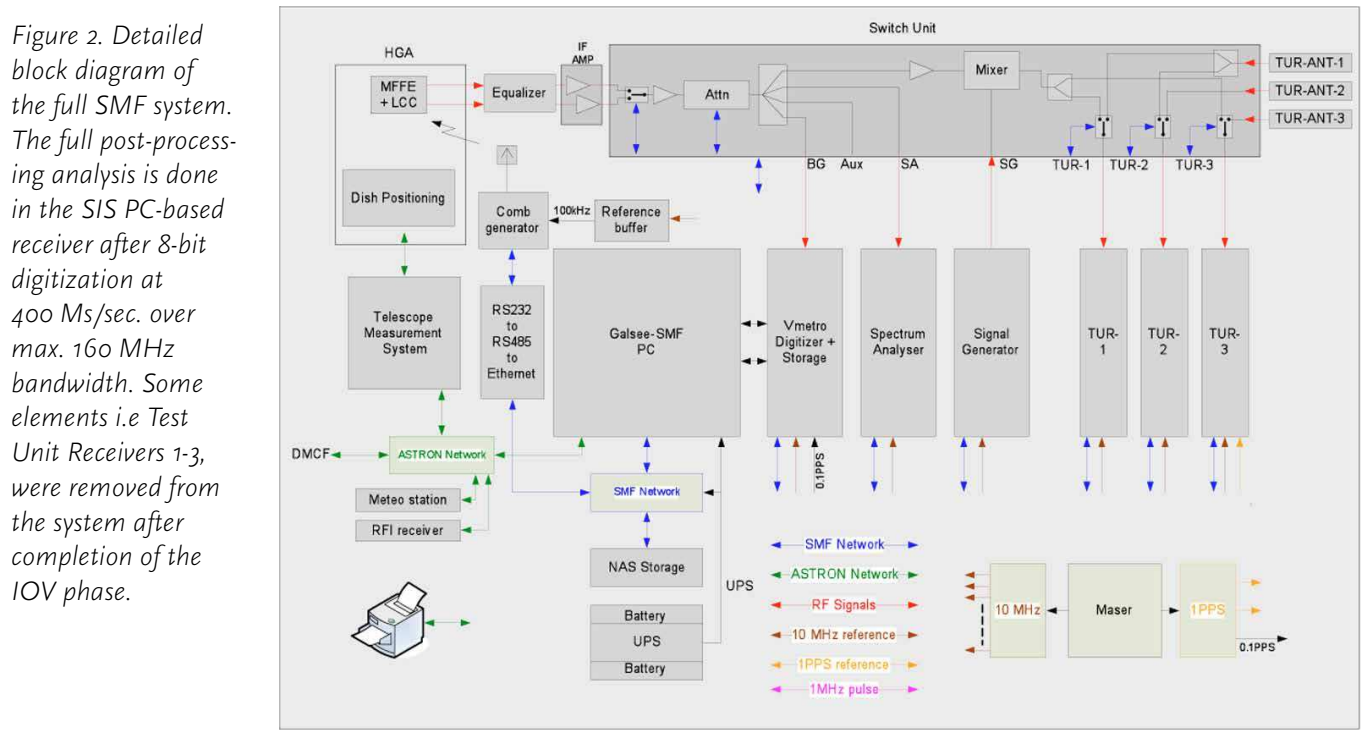

In the present system, the maximum observable bandwidth of $160 \mathrm{MHz}$ allows to simultaneously observe and analyse the Galileo specific E5+E6 (1145-1300 $\mathrm{MHz}$ ) bands and the E1 (1554-1596 MHz) band separately.
While the very low system noise is not strictly necessary, its proven advantage is the very high dynamic range observation of the Galileo spectra to allow analysing the signal spectral purity to a high degree.

With some sensitivity compromise, a new uncooled and dedicated wideband receiver is now being developed, allowing for the coherent observation of all Galileo bands besides those of other, GNSS satellites, in one go. This dedicated wideband receiver makes use of a special frequency mixing scheme to gain experience in coherent spectra reception and data analysis without affecting the digital back-end. A next receiver architecture will be designed such that the complete $500 \mathrm{MHz}$ frequency band can be analyzed.

\section{Gain calibration}

The receiver antenna pattern is calibrated using holographic measurements on radio astronomical sources. From these measurements, the antenna pattern is characterized in detail from which the efficiency and pointing errors are determined.

The absolute gain of the receiver chain is calibrated using strong astronomica radio sources of which the spectral power flux density at the Earth is accurately known. Measurements have shown that the stability of the gain of the receiver chain is better than $0.06 \mathrm{~dB}$ over a period of 8 hours.

\section{Phase calibration}

As the WSRT is usually used as an interferometer, it has been demonstrated that the phase stability (obtained from astronomical measurements) is better than \pm 4 degrees over a period of 12 hours. However, due to the fact that al telescopes in the interferometer have the same properties, it is not possible to use these measurements to calibrate the frequency-dependent group delay or phase properties of the receiver chain.

The method used for the frequency dependent broadband phase calibration is described in detail in "J. van der Marel and A. Bos, "Broadband Phase Calibration of a High Gain Antenna", Proc. of the 32nd ESA Workshop on Antennas for Space Applications, October 2010". A summary from this is given here.

The impulse response of a filter or a receiver chain is the Fourier transform of the transfer function of the filter or receiver chain. The spectrum of a pulse is wide and its phase is well defined. Therefore, pulses are very well suited for the calibration of a receiver chain with a large instantaneous bandwidth. Frequency conversions in the receiver chain do not have an impact on the analysis. A repetitive pulse generator, or comb generator, can be used for accurate pass-band calibration, both in amplitude and phase. The signal is transmitted from near to the apex of the telescope and synchronized to the H-maser and subsequently received by the modified frontend in the focus. 
The picture shows the telescope with the mounted Galileo receiver. The insert shows the wideband spiral antenna close to the apex from which the pulse train is transmitted for reception in the receiver.

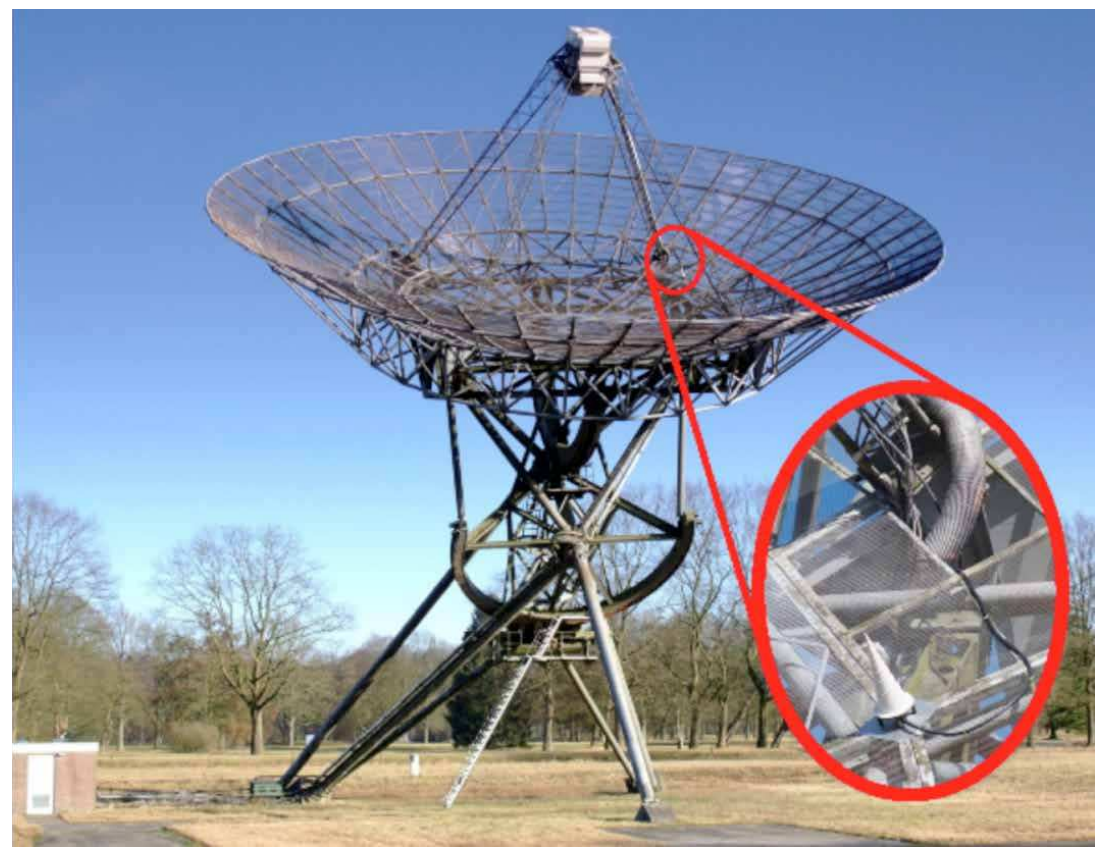

In the ideal case the periodic signal consists of a repetition of Dirac delta pulses but in practise have a finite bandwidth, thus producing an impulse with a finite length. It is shown in [5] that the inverse of the transfer function can be computed from which a deconvolution filter can be determined. From this a highly accurate time delay response function can be derived. Together with the installed noise calibration system and using radio source calibration, the accuracy with which the phase of the band pass can be determined is $\pm 0.3^{\circ}$ and for the amplitude an accuracy of better than $\pm 0.05 \mathrm{~dB}$ can be obtained. These numbers have been obtained during repeating measurements over a period of 8 hours, which shows that both the impulse generator and the receiver chain are very stable.

\section{Measurement analyses}

As described in the introduction of this paper, the analyses is performed by a software receiver schematically shown in Figure 4 . The received data is being sampled by a high-speed data sample, and the stored data is being processed to perform a number of analyses, including -but not restricted to-- the estimation of the Power Spectral Density (PSD) of the signal, the estimation of the Doppler-shift, signal demodulation, power estimation, and the constellation diagram.

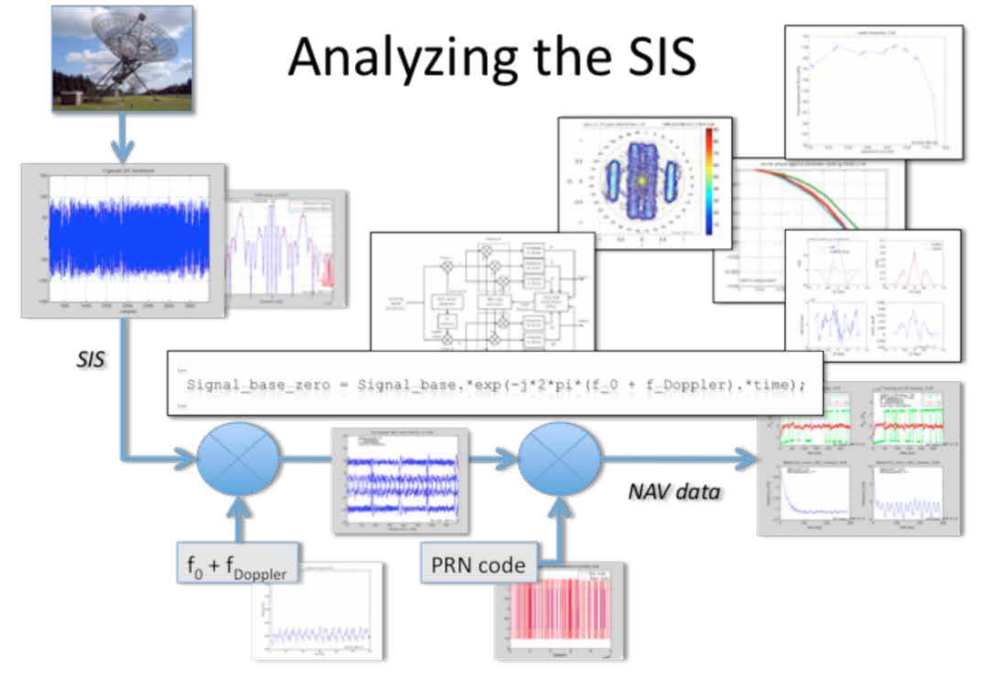

An example the PSD of the E1 signal of Galileo is given in the following figure 5 Some additional analyses are being made as well, such as the comparison with the theoretical PSD, and the estimation of imbalances in the PSD by comparing the amplitude of the main lobes of the PSD.

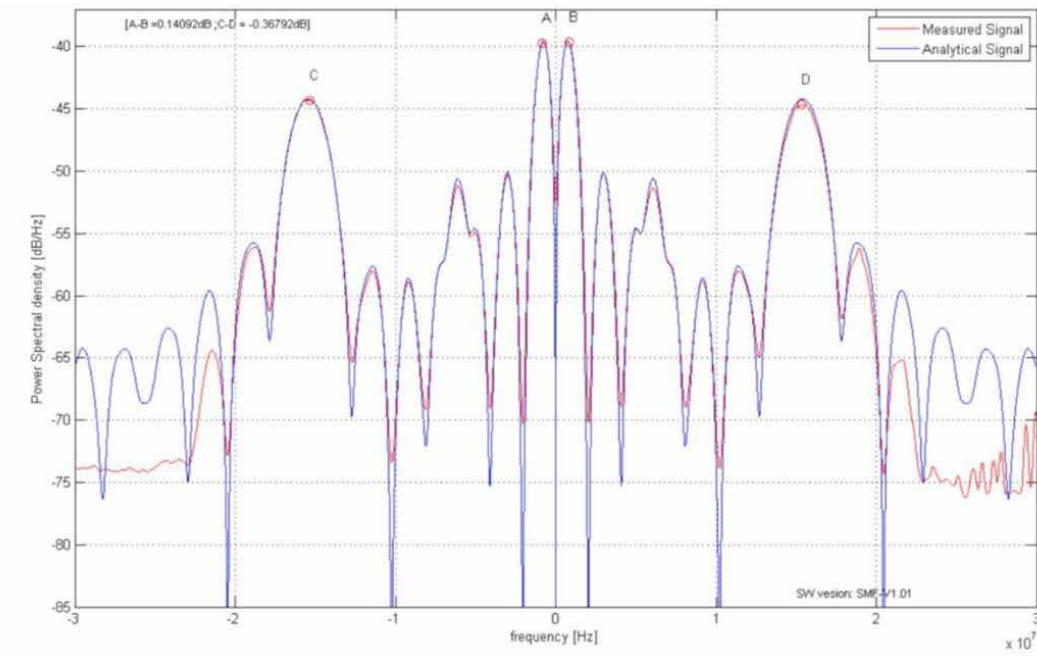

Summary

We demonstrated that with adequate modifications including dedicated phase and amplitude calibration and the development of the Signal in Space receiver, a radio telescope of the Westerbork array could successfully be employed for high performance GNSS monitoring. With some sensitivity compromise, the development of a wider band uncooled digital receiver instead of the relatively complicated Multi Frequency Front End, holds promise for a highly flexible next step. 


\section{Discovery of Heaven in Westerbork

In September 2000 the WSRT was the scene of the movie "Discovery of Heaven" based on the Harry Mulish novel "De ontdekking van de hemel", and produced by Jeroen Krabbé, Edwin de Vries and Ate de long. Hosting a complete film crew is something we underestimated a bit. They literally took over the entire site! But it gave us a lot joy and to be fair, we had to close dow the WSRT for a couple of weeks anyway because of major infrastruc ture work.

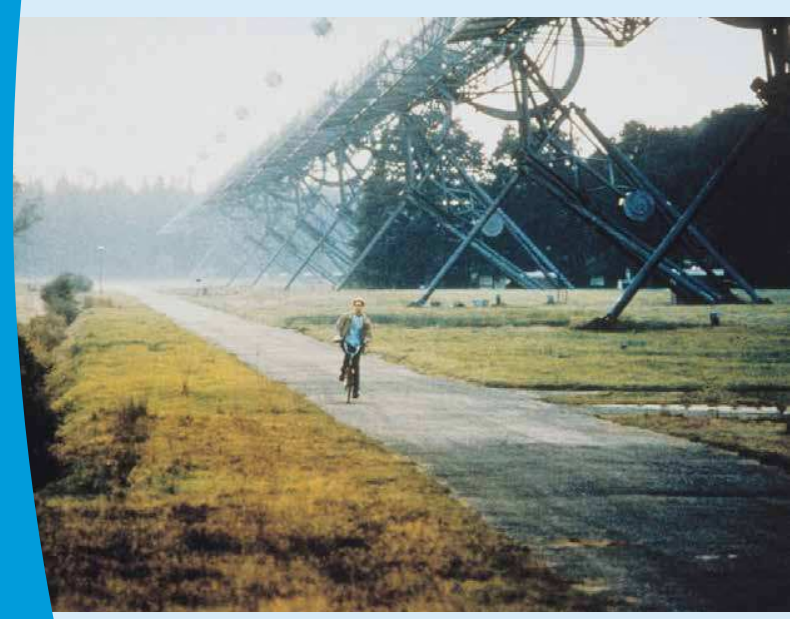

Figure 1: Scene from It all started in spring 2000. Jeroen the movie. Quinten Krabbé and Edwin de Vries visited is biking along the the WSRT, looking for possible plac telescopes. es to shoot the movie. They loved the place! Of course, Jan Pieter gave them "the full tour", so they concluded that a major part of the movie must be recorded in Westerbork. I do not know if this tour was the reason for changing the script, but the original Dwingeloo parts in the book by Harry Mulish were recorded in Westerbork as well.
wThe production team arrived in September 2000 to shoot the first scenes. In the construction hall "little Hollywood" arose, including campers for the lead movie actors, Stephen Fry, Greg Wise, Flora Montgomery and Neil Newbon, and full catering service, etc.

Making a movie requires a lot of socalled 'takes' and scenes sometimes had to be redone over ten times. One of the scenes included a car accident during which a tree would fall To make multiple takes possible, the tree was equipped with a hinge - a klap-boom in Dutch. They shot this scene in the middle of the night and they needed some ASTRON people to assist them. Rob Millenaar, Harm-Jan Stiepel and myself were there the whole time!

A somewhat expensive mistake was mixed up. The producers wanted an overview shot in which a car arrives at the telescopes. Drones were not yet available in 2000 so one of the biggest cranes available was rented. It was a beautiful shot, but when Jeroen Krabbé saw it, he was unhappy. They had used the wrong car! The 1970 car was used for the 1980 s shot. The next day, the crane was rented again and the shot redone, but this time with the correct car.

One of the most difficult "takes", was the arrival at the telescope during rainfall. Lots of rain was needed in the script. However it was not raining at all! It was a down on the road and hit the car. made when two time periods were

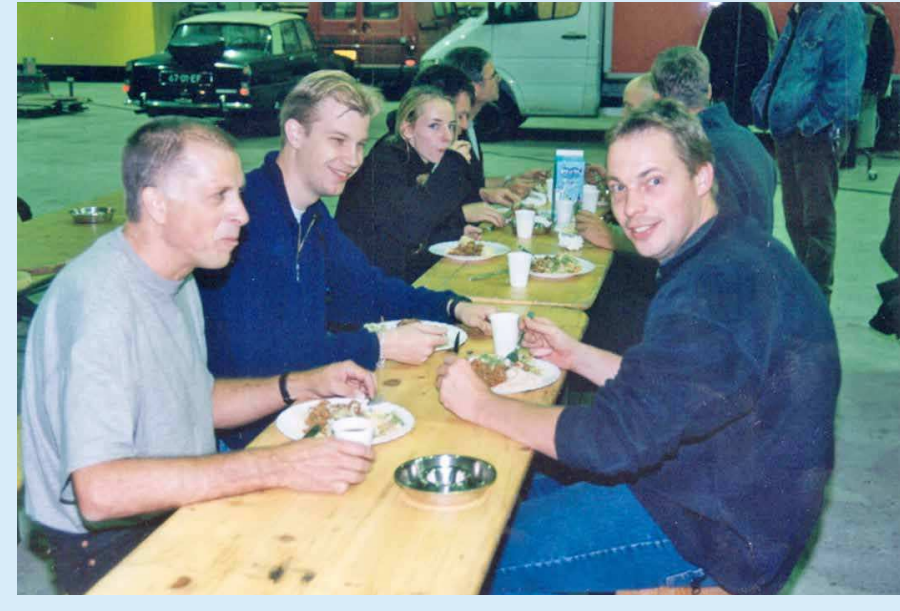

Figure 2: Rob Millenaar and Mark Bentum enjoying the full catering service of the film crew.

beautiful night at the WSRT so the technical crew created rain. Since we do not have a huge amount of we do not have a huge amount of water avallable at the telescopes, the crew rented two big trucks of water. At night time, the illuminated telescopes sprayed with water created a beautiful scene! (see Figure 3 thanks to Harm Jan Stiepel.)

In one of the scenes, Max Delius (the astronomer) had a discussion with one of his colleagues in the observing room. There was a black board in the room and Jeroen Krabbé asked us to put some "intelligent stuff" on it. This was our chance to
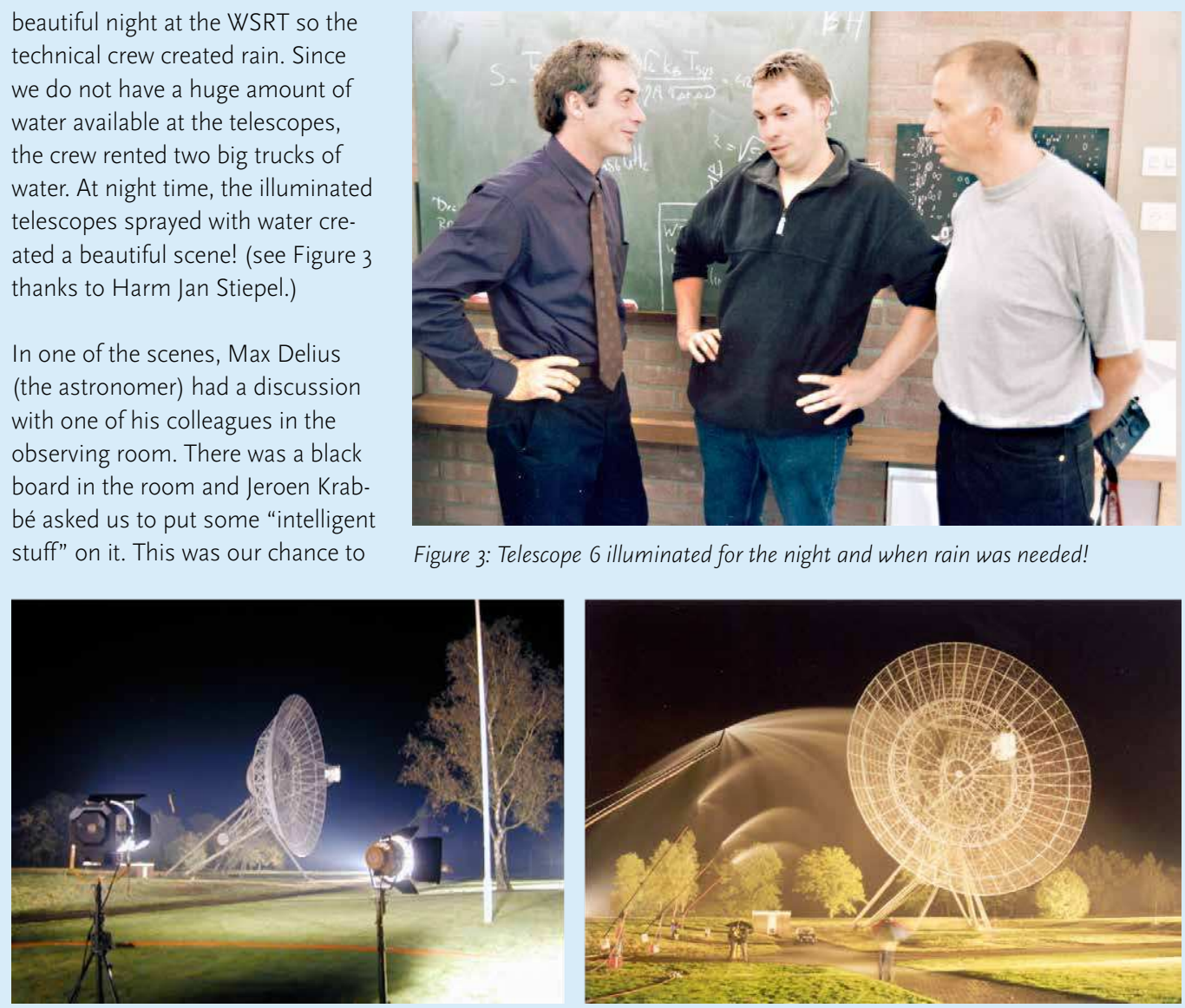

Figure 4: The actor Greg Wise (Max Delius) with Mark Bentum and Rob Millenaar before the black board with "intelligent equations".

ourselves (of course not telling (eroen). We used "intelligent" equations, but with our own initials, and with our kids initials, and making 42 the answer which readers of the "Hitchhikers guide to to the question of to the question of Life, the Universe,

ASTRON and IIVE personnel got to see the movie before it was released in a special showing just for us in the movie theatre in Hoogeveen in October 2001. We were about 200 people really enjoying it. The movie is, of course, still available. Look for all scenes with the WSRT in it!

CHAPTER 17.4 DISCOVERY OF HEAVEN IN WESTERBORK 
